\title{
Partial synchronization on a network with different classes of oscillators
}

\author{
Emmanuel Gräve de Oliveira* and Thomas Braun ${ }^{\dagger}$ \\ Instituto de Física, Universidade Federal do Rio Grande do Sul, Caixa Postal 15051, \\ 91501-970 Porto Alegre, Rio Grande do Sul, Brazil
}

(Received 24 September 2007; published 5 December 2007)

\begin{abstract}
Complete and partial synchronization have been largely studied on networks of identical coupled oscillators. However, we study a network in which not all oscillators when uncoupled show the same dynamics and nonetheless the network shows partial synchronization. Our system is composed by four Rössler oscillators diffusively coupled in a ring. Oscillators 1 and 3 are identical, as 2 and 4 are also. In short, the network is said to be composed of different classes of oscillators (in our example, two classes with two oscillators each). Primary synchronization is defined as the case when all oscillators on the same class are identically synchronized, for all classes. Secondary synchronization is related to the other possible cases of partial synchronization. Both are achieved for the system we have chosen, shown by means of direct integration and transverse Lyapunov exponent computation. Furthermore, evidence of riddled basins of attraction is presented.
\end{abstract}

DOI: 10.1103/PhysRevE.76.067201

PACS number(s): 05.45.Xt

Networks of coupled identical oscillators can show complete and partial synchronization [1-6]. On these networks, complete synchronization arises when all the systems present the same behavior, i.e., every pair of oscillators is identically synchronized. Partial synchronization occurs when some pairs of oscillators (but not all) are identically synchronized. In this work we study a network in which not all oscillators have the same dynamics when uncoupled. Our system is composed by four Rössler [7] oscillators diffusively coupled in a ring. Oscillators 1 and 3 are identical, as 2 and 4 are also. The system is given by the following equations $\left(\mathbf{x}^{(i+4)}=\mathbf{x}^{(i)}\right)$ :

$$
\dot{\mathbf{x}}^{(i)}=\mathbf{f}^{(i)}\left(\mathbf{x}^{(i)}\right)+c\left(\mathbf{x}^{(i+1)}+\mathbf{x}^{(i-1)}-2 \mathbf{x}^{(i)}\right),
$$

with $\quad \mathbf{x}^{(j)}=\mathbf{x}^{(j+n)}, \quad \mathbf{f}^{(1)}(\cdots)=\mathbf{f}^{(3)}(\cdots) \neq \mathbf{f}^{(2)}(\cdots)=\mathbf{f}^{(4)}(\cdots)$, and coupling parameter $c$. We also specify each Rössler oscillator to be

$$
\mathbf{f}^{(i)}\left(\mathbf{x}^{(i)}\right)=\left[\begin{array}{c}
-x_{2}^{(i)}-x_{3}^{(i)} \\
x_{1}^{(i)}+a_{i} x_{2}^{(i)} \\
b_{i}+x_{3}^{(i)}\left(x_{1}^{(i)}-c_{i}\right),
\end{array}\right]
$$

with parameters: $a_{i}=0.2, b_{i}=0.2$, and $c_{1}=c_{3}=10.0$, meanwhile $c_{2}=c_{4}=5.7$. Both types are chaotic oscillators with Lyapunov exponents $\lambda_{1} \approx 0.11$ and $\lambda_{2} \approx 0.071$. In short, we say that we have two different classes of oscillators. Although all of them are Rössler oscillators, we say that they belong to different classes because there is a big parameter mismatch among them and the two classes have very distinct Lyapunov exponents.

Our purpose is to investigate the identical synchronization [2] among identical oscillators on the network as the strength of the coupling $c$ is changed. Complete and partial synchronizations are related to the existence of invariant manifolds [3]. The system above does not possess an invari-

\footnotetext{
*emmanuel.deoliveira@ufrgs.br

†thomas.braun@ufrgs.br
}

ant manifold given by $\mathbf{x}^{(i)}=\mathbf{x}^{(j)}$ for all $i$ and $j$. This means that the system cannot show complete synchronization, since if $\mathbf{x}^{(i)}\left(t_{0}\right)=\mathbf{x}^{(j)}\left(t_{0}\right)$ for all $i$ and $j$ and for some $t_{0}$, then for some $t>t_{0}$ and some $i \neq j, \mathbf{x}^{(i)}(t) \neq \mathbf{x}^{(j)}(t)$ and the completely synchronized state is lost. Even so, the system above can have other invariant manifolds, related to partial synchronization. One can ask what kinds of partial synchronization (patterns) are allowed. For the example chosen, regarding partial synchronization, the invariant manifolds are the following:

(i) the $A B A B$ invariant manifold of synchronization $\mathbf{x}^{(1)}=\mathbf{x}^{(3)}$ and $\mathbf{x}^{(2)}=\mathbf{x}^{(4)}(A B A B$ pattern $)$;

(ii) the $A B C B$ invariant manifold of synchronization $\mathbf{x}^{(1)}$, $\mathbf{x}^{(2)}=\mathbf{x}^{(4)}$, and $\mathbf{x}^{(3)}(A B C B$ pattern $)$;

(iii) the $A B A C$ invariant manifold of synchronization $\mathbf{x}^{(1)}=\mathbf{x}^{(3)}, \mathbf{x}^{(2)}$, and $\mathbf{x}^{(4)}$ (ABAC pattern).

Also, the whole space can be viewed as the invariant manifold for the desynchronized motion (referred to as the $A B C D$ pattern).

On networks of identical oscillators, the $A A A A$ pattern (for an example with $n=4$ ), related to complete synchronization, played an important role, since it is the pattern with the simplest spatial structure. Our example cannot show this pattern, but clearly the $A B A B$ is of special interest, since it represents the identical synchronization among all oscillators in the same class, for all classes present in the network. Therefore, we will call the $A B A B$ pattern as the primary pattern, in contrast with the $A B C B$ and $A B A C$ secondary patterns. It is interesting to note that the $A B A B$ pattern preserves the symmetries of the network. The network remains the same if we exchange oscillators 1 and 3 or 2 and 4 or both pairs. So does the $A B A B$ pattern, but the $A B C B$ and $A B A C$ patterns spontaneously break one of these symmetries, as also observed in networks of identical oscillators [3].

We begin with the primary synchronization, characteristic of the $A B A B$ pattern. Chaotic oscillators have sensitive dependence on initial conditions. If the oscillators are uncoupled, and the variables of the first oscillator are slightly different from the respective variables of the third oscillator, we should expect that the modulo of the difference between 
the respective variables of the oscillators would increase exponentially. The same happens to small differences between the second and the fourth oscillators. Then, small amounts of noise would drive the system away from the pattern $A B A B$. In order to characterize synchronization, these small differences are required to decrease exponentially. This criterion produces what is called a weak synchronization [8], in which the synchronized state is an attractor in the sense of Milnor [9]. Strong synchronization [8] also requires the Lyapunov stability of the attractor, and will not be studied here.

We remind that since we are studying oscillators, the synchronized state must be oscillatory. Defining the Lyapunov exponent of the synchronized state $\lambda$, the cases of partial synchronization are restricted to $\lambda \geq 0$. Adapting the procedure used in previous works [1-3], transverse Lyapunov exponents are used to identify synchronization. The first step is to recognize that the dynamics of the synchronized state can be fully described by the equations

$$
\begin{aligned}
& \dot{\mathbf{s}}^{(1)}=\mathbf{f}^{(1)}\left(\mathbf{s}^{(1)}\right)+2 c\left(\mathbf{s}^{(2)}-\mathbf{s}^{(1)}\right), \\
& \dot{\mathbf{s}}^{(2)}=\mathbf{f}^{(2)}\left(\mathbf{s}^{(2)}\right)+2 c\left(\mathbf{s}^{(1)}-\mathbf{s}^{(2)}\right),
\end{aligned}
$$

where $\mathbf{s}^{(1)}=\mathbf{x}^{(1)}=\mathbf{x}^{(3)}$ and $\mathbf{s}^{(2)}=\mathbf{x}^{(2)}=\mathbf{x}^{(4)}$. These equations show that the synchronized state depends on $c$, different from the case of complete synchronization. Let $i=1,2,3,4$ and $\mathbf{x}^{(i)}=\mathbf{s}^{\left(\eta_{i}\right)}+\delta \mathbf{x}^{(i)}$, where $\eta_{i}=1$ if $i=1,3$ and $\eta_{i}=2$ if $i$ $=2,4$. Then, the system linearized around the synchronized state can be expressed as

$$
\delta \dot{\mathbf{x}}^{(i)}=D \mathbf{f}^{(i)}\left(\mathbf{s}^{\left(\eta_{i}\right)}\right) \delta \mathbf{x}^{(i)}+c\left(\delta \mathbf{x}^{(i+1)}+\delta \mathbf{x}^{(i-1)}-2 \delta \mathbf{x}^{(i)}\right) .
$$

The evolution of the differences $\delta \mathbf{y}^{(1)}=\delta \mathbf{x}^{(1)}-\delta \mathbf{x}^{(3)}$ and $\delta \mathbf{y}^{(2)}=\delta \mathbf{x}^{(2)}-\delta \mathbf{x}^{(4)}$ is our main focus now. If $\delta \mathbf{y}^{(1)}$ and $\delta \mathbf{y}^{(2)}$ vanish exponentially fast for $t \rightarrow \infty$, the network is said to achieve primary synchronization. Writing the equations for $\delta \dot{\mathbf{y}}^{(i)}$, one has $(i=1,2)$,

$$
\delta \dot{\mathbf{y}}^{(i)}=D \mathbf{f}^{(i)}\left(\mathbf{s}^{\left(\eta_{i}\right)}\right) \delta \mathbf{y}^{(i)}-2 c \delta \mathbf{y}^{(i)} .
$$

The transverse Lyapunov exponent is defined as

$$
\lambda_{\perp}=\lim _{t \rightarrow \infty} \frac{1}{t} \ln \frac{\delta(t)}{\delta(0)},
$$

where $\delta(t)=\sqrt{\sum_{i}\left[\delta \mathbf{y}^{(i)}(t)\right]^{2}}$. If $\lambda_{\perp}<0$, then the small differences between oscillators 1 and $3\left[\delta \mathbf{y}^{(1)}(t)\right]$ and between oscillators 2 and $4\left[\delta \mathbf{y}^{(2)}(t)\right]$ decrease exponentially fast and initial conditions near to the manifold converge to it.

In Fig. 1 we have numerically calculated both the transverse and the synchronized state Lyapunov exponents from $c=0$ to $c=0.1$. The dynamics on the manifold of synchronization is found to be chaotic except in the periodic window $0.077 \gtrsim c \gtrsim 0.079$. For $c \approx 0.055$ or greater, the system primarily synchronizes. The minimum value of $c$ satisfying this condition is called $c_{\mathrm{s}}$. Thus, we have shown that in our example primary synchronization is possible, at least under a transverse Lyapunov exponent analysis.

Based on both the definition of the transverse Lyapunov exponent and on Eqs. (3a), (3b), and (5), it is easy to see that if $c=0$, then the transverse Lyapunov exponent is the Lyapunov exponent of the system (3a) and (3b), or simply

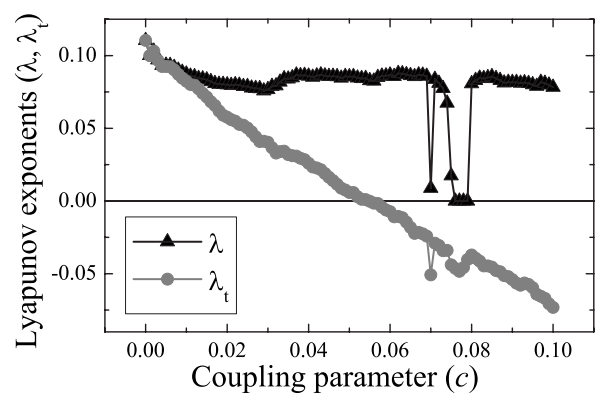

FIG. 1. The Lyapunov exponents related to the $A B A B$ pattern. Both exponents have the same value at $c=0$ and the transverse Lyapunov exponent decreases as an approximately affine function of $c$. For $c \gtrsim 0.055$, the system achieves primary synchronization.

$\lambda_{1}$. Synchronization and the variation of $\lambda_{\perp}$ with $c$ can be better understood with the help of the substitution $\delta \mathbf{y}^{(i)}$ $=e^{-2 c t} \delta \mathbf{y}^{(i)^{\prime}}$, that leads to the modified equation

$$
\delta \dot{\mathbf{y}}^{(i)^{\prime}}=D \mathbf{f}^{(i)}\left(\mathbf{s}^{\left(\eta_{i}\right)}\right) \delta \mathbf{y}^{(i)^{\prime}},
$$

and the correspondent transverse Lyapunov exponent

$$
\lambda_{\perp}=-2 c+\lim _{t \rightarrow \infty} \frac{1}{t} \ln \frac{\delta^{\prime}(t)}{\delta^{\prime}(0)}=-2 c+\lambda_{\perp}^{\prime},
$$

where $\delta^{\prime}(t)=\sqrt{\sum_{i}\left[\delta \mathbf{y}^{(i)^{\prime}}(t)\right]^{2}}$. Clearly, if $\lambda_{\perp}^{\prime}$ remains bounded as $c \rightarrow \infty$ the system achieves primary synchronization. It will happen when the dynamics given by Eqs. (3a) and (3b) has a limit attractor for $c \rightarrow \infty$. If the oscillators are somehow equivalent and the coupling has the tendency of making the difference between these attractors smaller, one can expect that this limit exists.

Studying both $A B C B$ and $A B A C$ patterns, different features (from the $A B A B$ pattern) are remarkable. In these patterns, two identical oscillators have distinct evolutions. This spatial ordering is originated by a symmetry breaking [10]. This can happen only if the initial conditions are different, or the system is subjected to noise, which implies changing the dynamics of the system. Although computer simulations are used and some noise is introduced by rounding errors, the spatial ordering is attributed to the random choosing of the initial conditions.

One might expect that the $A B C B$ pattern would be easier to find, since it implies the identical synchronization of oscillators that have smaller Lyapunov exponents when uncoupled and it is known that the system is going to show the $A B A B$ pattern for large $c$. Although counterintuitive, the opposite is obtained below. This result comes from the fact that the oscillators are not weakly coupled, meaning that we cannot consider them independently. The influence from different oscillators changes the dynamics of each oscillator as the coupling grows and the system must be considered as a whole.

For the $A B C B$ pattern dynamics we have

$$
\begin{gathered}
\dot{\mathbf{s}}^{(1)}=\mathbf{f}^{(1)}\left(\mathbf{s}^{(1)}\right)+2 c\left(\mathbf{s}^{(2)}-\mathbf{s}^{(1)}\right), \\
\dot{\mathbf{s}}^{(2)}=\mathbf{f}^{(2)}\left(\mathbf{s}^{(2)}\right)+c\left(\mathbf{s}^{(1)}+\mathbf{s}^{(3)}-2 \mathbf{s}^{(2)}\right),
\end{gathered}
$$




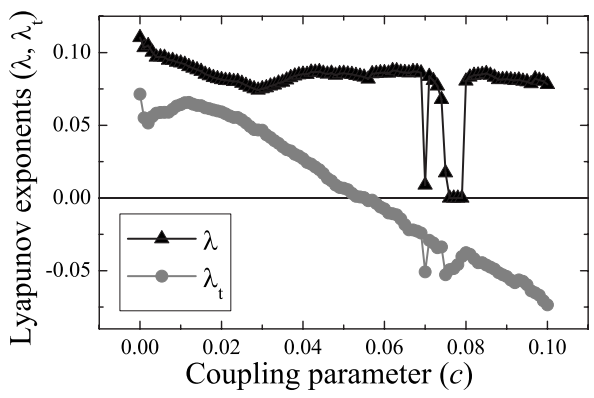

FIG. 2. The Lyapunov exponents related to the $A B C B$ pattern. The transverse Lyapunov exponent becomes negative for nearly the same range of $c$ on which the system achieves primary synchronization. In this case, the $A B C B$ pattern is degenerated to $A B A B$.

$$
\dot{\mathbf{s}}^{(3)}=\mathbf{f}^{(1)}\left(\mathbf{s}^{(3)}\right)+2 c\left(\mathbf{s}^{(2)}-\mathbf{s}^{(3)}\right),
$$

where $\mathbf{s}^{(1)}=\mathbf{x}^{(1)}, \mathbf{s}^{(2)}=\mathbf{x}^{(2)}=\mathbf{x}^{(4)}$, and $\mathbf{s}^{(3)}=\mathbf{x}^{(3)}$. Then we define the difference near the synchronized state as $\delta \mathbf{y}^{(1)}=\delta \mathbf{x}^{(2)}$ $-\delta \mathbf{x}^{(4)}$ and derive the equation

$$
\delta \dot{\mathbf{y}}^{(1)}=D \mathbf{f}^{(2)}\left(\mathbf{s}^{(2)}\right) \delta \mathbf{y}^{(1)}-2 c \delta \mathbf{y}^{(1)} .
$$

Then, the transverse Lyapunov exponent is defined as

$$
\lambda_{\perp}=\lim _{t \rightarrow \infty} \frac{1}{t} \ln \frac{\left\|\delta \mathbf{y}^{(1)}(t)\right\|}{\left\|\delta \mathbf{y}^{(1)}(0)\right\|} .
$$

In Fig. 2 the Lyapunov exponents related to the $A B C B$ pattern are shown. The transverse Lyapunov exponent becomes negative in the same range of $c$ on which the system achieves primary synchronization and there is no difference between the synchronized state exponents. In this case, the $A B C B$ pattern is degenerated [3] to $A B A B$. Thus the network cannot display the $A B C B$ pattern.

For the $A B A C$ pattern, the dynamics on the manifold of synchronization is given by

$$
\begin{gathered}
\dot{\mathbf{s}}^{(1)}=\mathbf{f}^{(1)}\left(\mathbf{s}^{(1)}\right)+c\left(\mathbf{s}^{(2)}+\mathbf{s}^{(3)}-2 \mathbf{s}^{(1)}\right), \\
\dot{\mathbf{s}}^{(2)}=\mathbf{f}^{(2)}\left(\mathbf{s}^{(2)}\right)+2 c\left(\mathbf{s}^{(1)}-\mathbf{s}^{(2)}\right),
\end{gathered}
$$

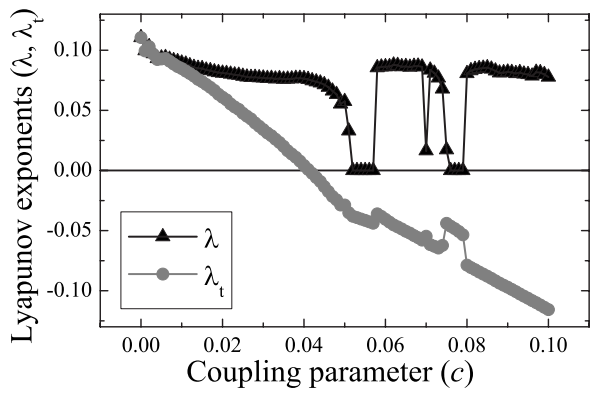

FIG. 3. The Lyapunov exponents related to the $A B A C$ pattern. Secondary synchronization is finally achieved for the range 0.042 $\lesssim c \lesssim 0.055$. The existence of two basins of attraction appears for $c \gtrsim 0.055$ between $A B A C$ and $A B A B$ patterns.

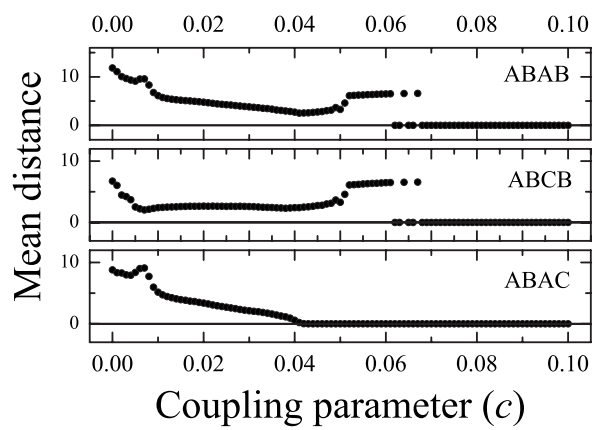

FIG. 4. The mean distance of the system dynamics from the synchronization manifolds is calculated varying $c$ for random initial conditions. The region of coexistence of $A B A B$ and $A B A C$ patterns is easily identified. The complete absence of $A B C B$ pattern confirms our early predictions.

$$
\dot{\mathbf{s}}^{(3)}=\mathbf{f}^{(2)}\left(\mathbf{s}^{(3)}\right)+2 c\left(\mathbf{s}^{(1)}-\mathbf{s}^{(3)}\right),
$$

where $\mathbf{s}^{(1)}=\mathbf{x}^{(1)}=\mathbf{x}^{(3)}, \mathbf{s}^{(2)}=\mathbf{x}^{(2)}$, and $\mathbf{s}^{(3)}=\mathbf{x}^{(4)}$. The evolution of the small difference $\delta \mathbf{y}^{(1)}=\delta \mathbf{x}^{(1)}-\delta \mathbf{x}^{(3)}$ is given by

$$
\delta \dot{\mathbf{y}}^{(1)}=D \mathbf{f}^{(1)}\left(\mathbf{s}^{(1)}\right) \delta \mathbf{y}^{(1)}-2 c \delta \mathbf{y}^{(1)} .
$$

The definition of a transverse Lyapunov exponent is the same as in the case of the $A B C B$ pattern.

In Fig. 3 the Lyapunov exponents are calculated as a function of $c$ for the $A B A C$ pattern. Secondary partial synchronization is finally achieved in the range $0.042 \leqq c \leqq 0.055$, since in this region we found only this pattern. For $c$ $\gtrsim 0.055$, one could think that only the $A B A B$ pattern exists. However, comparing with Fig. 1, it can be seen that there are traces of the coexistence of two attractors, since the Lyapunov exponent of the synchronized state is not the same for the $A B A B$ and $A B A C$ patterns. The $A B A C$ presents a pe-

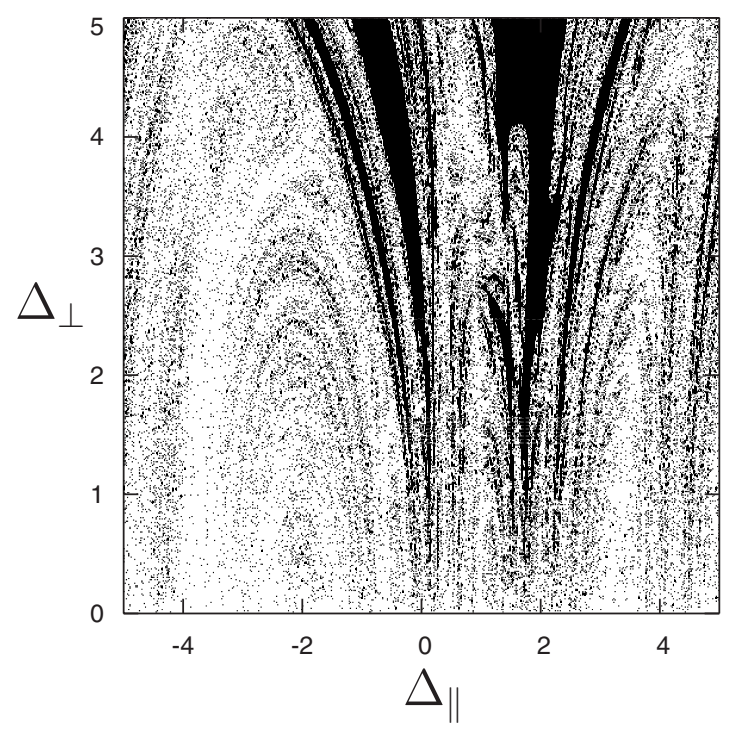

FIG. 5. Evidence of riddled basins of attraction at $c=0.62$. Black points represent initial conditions leading to $A B A C$ pattern, while white points lead to $A B A B$ pattern. $\Delta_{\|}\left(\Delta_{\perp}\right)$ is the parallel (perpendicular) component to the $A B A B$ manifold. 
riodic state, while the $A B A B$ presents a chaotic one, showing that the $A B A C$ pattern is not degenerated to the $A B A B$ pattern. However, for sufficiently large $c$, this discrepancy between the synchronized state Lyapunov exponents vanishes.

In Fig. 4 we see exactly as stated in the last paragraph. In this figure, we integrated our system for randomly chosen initial conditions and searched for patterns after the transient. We could not find the $A B C B$ pattern, in agreement with Fig. 2. Also, the coexistence of two patterns is shown for $c$ $\approx 0.065$. Within the framework of weak synchronization, this coexistence of different kinds of partial synchronization can be caused by the existence of riddled basins of attraction [11] in our system.

Figure 5 shows the evidence of riddled basins of attraction (based on the method developed in [11]) at $c=0.62$. Black (white) points represent initial conditions leading to the $A B A C(A B A B)$ pattern. The initial conditions used are $x^{(1)}(0)=x^{(3)}(0)=[8.62526,4.59168,12.5865]^{T}$, $x_{2,3}^{(2)}=x_{2,3}^{(4)}(0)=0.308883,7.13878$, and $x_{1}^{(2),(4)}(0)=-1.1612$ $+(1 / \sqrt{2})\left(\Delta_{\|} \mp \Delta_{\perp}\right)$. The quantity $\Delta_{\|}([-5,5]$, with increments of 0.02) changes the initial conditions on the manifold of synchronization, while $\Delta_{\perp}([0,5]$, with increments of 0.01$)$ measures how far from it they are. No matter how near the initial conditions are from the $A B A B$ manifold of synchronization, there are always initial conditions in the neighborhood that put the system in the $A B A C$ pattern. As clear evidence of riddled basins, we find the power law $20 \Delta_{\perp}^{0.49}$ for the percentage of the initial conditions leading to the $A B A C$ pattern.

In conclusion, we have a system with possibilities of identical synchronization, even not all oscillators being identical. We have shown that partial synchronization can arise on such a network, including the case in which the oscillators in the same class are identically synchronized. Also the case of spatial ordering is shown. Explanation about these facts are given through transverse Lyapunov exponents. We have shown the coexistence of attractors and that our system can present riddled basins of attraction. The strong synchronization of the system is left as an open question. In spite of using a specific example, we understand that a large group of systems can have almost the same features, differing only by a quantitative analysis.

The authors would like to acknowledge Roberto Paaz, Rodrigo Link Federizzi, and Leonardo Pletsch for interesting discussions. This work was partially supported by Brazilian agencies $\mathrm{CNPq}$ and PROPESQ-UFRGS.
[1] H. Fujisaka and T. Yamada, Prog. Theor. Phys. 69, 32 (1983).

[2] L. M. Pecora, T. L. Carrol, J. F. Heagy, G. A. Johnson, and D. J. Mar, Chaos 7, 520 (1997).

[3] Y. Zhang, G. Hu, H. A. Cerdeira, S. Chen, T. Braun, and Y. Yao, Phys. Rev. E 63, 026211 (2001).

[4] A. Pogromsky, G. Santoboni, and H. Nijmeijer, Physica D 172, 65 (2002).

[5] X. Li and G. Chen, IEEE Trans. Circuits Syst., I: Fundam. Theory Appl. 50, 1381 (2003).
[6] I. A. Heisler, T. Braun, Y. Zang, G. Hu, and H. A. Cerdeira, Chaos 13, 185 (2003).

[7] O. E. Rössler, Phys. Lett. A 57A, 397 (1976).

[8] M. Hasler and Y. Maistrenko, IEEE Trans. Circuits Syst., I: Fundam. Theory Appl. 44, 856 (1997).

[9] J. Milnor, Commun. Math. Phys. 99, 177 (1985).

[10] G. Hu, Y. Zhang, H. A. Cerdeira, and S. Chen, Phys. Rev. Lett. 85, 3377 (2000).

[11] E. Ott and J. C. Sommerer, Phys. Lett. A 188, 39 (1994). 